\title{
Correction to: Keystroke and Pointing Time Estimation for Touchscreen-Based Mobile Devices: Case Study Children with ASD
}

\author{
Angeles Quezada, Margarita Ramirez Ramírez, \\ Sergio Octavio Vázquez, Ricardo Rosales, Samantha Jiménez, \\ Maricela Sevilla, and Roberto Muñoz
}

\section{Correction to: \\ Chapter "Keystroke and Pointing Time Estimation for Touchscreen-Based Mobile Devices: Case Study Children with ASD" in: Á. Rocha et al. (Eds.): New Knowledge in Information Systems and Technologies, AISC 931, https://doi.org/10.1007/978-3-030-16184-2_74}

In the original version of the book, the chapter "Keystroke and Pointing Time Estimation for Touchscreen-Based Mobile Devices: Case Study Children with ASD” was published with six authors and now an author (Prof. Roberto Muñoz) has been added to this chapter. The correction chapter and the book is now updated with the change. 